\section{Kidney \\ Blood Pressure Research}

\title{
Barriers to Physical Activity in Chronic Hemodialysis Patients: A Single-Center Pilot Study in an Italian Dialysis Facility
}

\author{
Enrico Fiaccadori ${ }^{a}$ Alice Sabatino ${ }^{a}$ Franco Schito $^{b}$ Francesca Angella $^{c}$ \\ Martina Malagolic Marco Tuccic Adamasco Cupistid Alessandro Capitaninie \\ Giuseppe Regolistia
}

aRenal Pathophysiology Unit, Clinical and Experimental Medicine Department, University of Parma; ${ }^{b}$ Nephrology and Dialysis Unit, ASL Parma; 'Faculty of Health and Sport Sciences, Parma University, Parma; ${ }^{d}$ Department of Clinical and Experimental Medicine, University of Pisa, Pisa; eNephrology and Dialysis Unit, ASL Pistoia, Italy

\section{Key Words}

Hemodialysis • End-stage renal disease • Exercise • Physical activity

\begin{abstract}
Background/Aims: In patients on chronic dialysis a sedentary lifestyle is a strong, yet potentially modifiable, predictor of mortality. The present single-center pilot study evaluated social, psychological and clinical barriers that may hinder physical activity in this population. Methods: We explored the association between barriers to physical activity and sedentarism in adult patients at a chronic dialysis facility in Parma, Italy. We used different questionnaries exploring participation in physical activity, physical functioning, patient attitudes and preferences, and barriers to physical activity perceived by either patients or dialysis doctors and nurses. Results: We enrolled 104 patients, (67 males, 65\%), mean age 69 years (79\% of patients older than 60 years); median dialysis vintage 60 months (range 8-440); mean Charlson score 5.55, ADL (Activities of Daily Living) score 5.5. Ninety-two participants (88.5\%) reported at least one barrier to physical activity. At multivariable analysis, after adjusting for age and sex, feeling to have too many medical problems (OR 2.99, 95\% CI 1.27 to 7.07 ; $P=0.012$ ), chest pain (OR 10.78, 95\% CI 1.28 to $90.28 ; \mathrm{P}=0.029)$ and sadness (OR 2.59, 95\% CI 1.10 to 6.09; $P=0.030$ ) were independently associated with physical inactivity. Lack of time for exercise counseling and the firm belief about low compliance/interest by the patients toward exercise were the most frequent barriers reported by doctors and nurses. Conclusion: We identified a number of patient-related and health personnel-related barriers to physical activity in patients on chronic dialysis. Solutions for these barriers should be addressed in future studies aimed at increasing the level of physical activity in this population.
\end{abstract}




\section{Kidney Blood Pressure Research}

Fiaccadori/Sabatino/Schito et al.: Barriers to Physical Activity in Hemodialysis Patients

\section{Introduction}

Physical activity is significantly reduced in patients on hemodialysis, regardless of age, and is low even when compared to age-matched sedentary populations [1].

In the clinical setting of End-Stage Renal Disease (ESRD) low levels of physical activity may significantly contribute both to frailty/disability, by reducing physical performance, and to protein energy wasting, by favouring skeletal muscle atrophy [2]. Moreover, impaired mobility and reduced physical function have been demonstrated to set the stage for further increases of an already high cardiovascular mortality risk [3-4].

Few data are currently available on factors contributing to a sedentary lifestyle in patients on chronic hemodialysis. As documented in two recent studies from the US [5] and China [6], it is likely that a complex mix of disease- and patient-specific reasons (socioeconomical, psychological, cultural, and clinical) play a key role in determining physical inactivity. Moreover, also health personnel (dialysis doctors and nurses) attitudes could significantly contribute to sedentarism in dialysis patients, since low levels of exercise counseling have been documented among nephrologists [7].

No data are currently available on this topic in European dialysis patient populations. Thus, the present single-center pilot study was aimed at exploring barriers to physical activity in a cohort of patients from an Italian dialysis facility.

\section{Patients and Methods}

After providing informed consent, all adult subjects on routine hemodialysis for at least 6 months at the National Health System Chronic Dialysis Facility of Parma (Nefrologia e Dialisi, Azienda Sanitaria Parma, Italy) were recruited. The study was approved by the Institutional Review Board of Parma. Demographic data, routine laboratory parameters, comorbidities and data on dialysis treatment and vascular access were extracted from the electronic records. Total comorbidity burden was evaluated by the Charlson index, and the general performance status was summarized by the Karnofski score.

Questionnaires administered to the patients during the dialysis session, and to dialysis doctors and nurses covered the areas of interest for the study purpose. Questionnaires for the patients were related to levels of mobility/autonomy and physical functioning, self-reported physical activity, quality of life, and perceived barriers to physical activity. In particular, the level of self-reported physical functioning/ autonomy was evaluated by the Katz Independence in daily living questionnaire (ADL, Activities of Daily Living questionnaire), a self-reported test on mobility and performance based on 18 items describing different levels of autonomy in physical activity related to participation in life activities [8].

The level of physical activity was derived from the 94-item Human Activity Profile (HAP) [9], which includes the assessment of activities across a wide range of energy requirements. On the HAP, activity items are ranked based on their estimated energy expenditure, from the lowest to the highest. For example, getting in and out of chairs or bed without assistance (item 10), is the activity requiring the least energy expenditure; while running or jogging 3 miles in $\leq 30$ minutes (item 89), is the activity requiring the greatest energy expenditure. For each item, the respondent indicates whether he or she is still doing, has stopped doing, or never did the activity in question. The HAP items were used to derive the MAS (Maximum Activity Score) and the AAS (Adjusted Activity Score). The MAS is the highest oxygen-demanding activity (highest item number) that the respondent is able to perform. The AAS is obtained by subtracting from the MAS the total number of activities the respondent has stopped doing that have numbers lower than the MAS of that patient. The AAS is considered as a measure of usual physical activity level. MAS and AAS score results were compared to established normative data by age and gender [9].

Patient-related barriers to physical activity were evaluated on the basis of the questionnaire by Delgado \& Johansen [5], which includes questions related to different categories of disease- and patientspecific barriers to physical activity: psychological barriers, physical barriers, economical barriers, lack of time and comorbidities. Participants were classified as having endorsed a barrier if they reported that they 'sometimes', 'often' or 'always' experienced that barrier; participants who reported that they 'never' experienced a barrier were classified as not endorsing that barrier. 


\section{Kidney \\ Blood Pressure Research}

A slight modification of the Johansen's questionnaire on personal attitude to physical activity counseling [7] was used for nephrologists and dialysis nurses. The applied questionnaire consisted of 17 questions (13 in the case of nurses) regarding dialysis personnel opinions and practices related to exercise counseling, such as for example: "How often do you assess patients' physical activity levels?" "Do you advise sedentary patients to increase activity?" "Do you provide patients with a specific exercise prescription?" "Do you offer written information about physical activity to patients?" "Do you refer patients to physical activity specialists?" "Does your facility provide exercise equipment for use in the dialysis unit?" Doctors and nurses were also asked to indicate their level of agreement or disagreement with a number of statements related to potential barriers to exercise counseling.

\section{Statistical analysis}

Descriptive statistics is given as Mean and standard deviation (SD) or median with 25-75th percentile for continuous variables according to their normal or non-normal distribution, respectively; frequencies (percentage) are presented for categorical variables. The level of physical activity was categorized according to the AAS (<53 sedentary, 53-74 moderately active, > 74 active); subsequently, the degree of physical activity was dichotomized (i.e., inactive vs active) by grouping together moderately active and active patients. The association between the degree of, and each perceived barrier to, physical activity was tested by chi-square and univariate logistic regression. Barriers selected as being significantly associated with the degree of physical activity at univariate analysis were entered in a multivariable logistic stepwise regression model to investigate which barriers were associated independently with physical inactivity after adjustment for age and sex. A two-tailed $P$ value of less than 0.05 was considered statistically significant. All statistical analyses were performed using SPSS v.21.

\section{Results}

\section{Demographic and clinical characteristics}

One-hundred four patients participated in the study. Most of them (67 patients, 65\%) were males, mean age was 69 years, with $79 \%$ of patients being older than 60 years. Only 27 patients $(26 \%)$ had a high education level. Median dialysis vintage was 60 months (range 8-440); most of the patients were dialyzed through an arterovenous fistula, thrice weekly; 84 patients $(81 \%)$ had hemoglobin levels $<12 \mathrm{~g} / \mathrm{dL}$, and half of the population $(47 / 104$, $45 \%)$ had high serum C-reactive protein levels. Ischemic heart disease (36\%) and diabetes mellitus (31\%) were the most frequent chronic comorbidities; mean Charlson score was 7.3, mean ADL score was 5.5.

\section{Physical activity levels}

On the basis of the MAS and AAS components of the Human Activity Profile ESRD patients were very sedentary (Table 1). Both MAS and AAS values were significantly decreased compared with reference healthy subjects: even taking into account the high mean age of dialysis patients, MAS and AAS values were below the $25^{\circ} / 5^{\circ}$ percentile of the normative healthy population in $40 \%$ / 30\% and in $80 \%$ / $66 \%$ of our patients, respectively. On the basis of AAS, more than half of the patients $(54 / 104,52 \%)$ could be classified as inactive, $30 \%(31 / 104)$ as moderately active, and only 19 (18\%) could be defined as active (Table 1).

\section{Barriers to physical activity}

Only 4 patients (3.8\%) reported no barriers at all. The prevalence of specific barriers to physical activity among patients is illustrated in Table 2 . Three percent had one barrier, $34 \%$ 2-4 barriers, $47 \%$ 5-9 barriers; 17 patients (16\%) indicated more than 10 barriers. The most frequently reported barriers were represented by fatigue in dialysis days (56.7\%), perception of too many medical problems (54.8\%), sadness (50\%), lack of motivation (42.3\%) and lack of time on dialysis days (32.7). Inactive subjects showed a higher prevalence of barriers for at least six of the barriers explored; at univariate analysis, barriers associated with inactivity 


\section{Kidney \\ Blood Pressure Research}

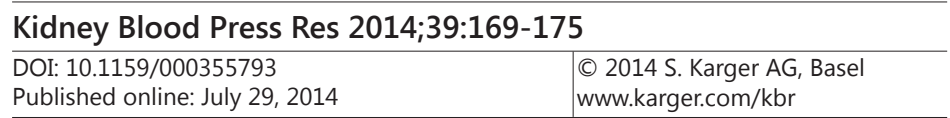

Table 1. Physical activity on the basis of the Human Activity Profile

\begin{tabular}{cccc}
\hline & $\begin{array}{c}\text { Patients on dialysis } \\
(\mathrm{n}=104)\end{array}$ & $\begin{array}{c}\text { Elderly subjects } \\
\text { age } 60-88 \text { yrs } \\
(\mathrm{n}=102) \S\end{array}$ & $\begin{array}{c}\text { Healthy subjects } \\
\text { age 20-79 yrs } \\
(\mathrm{n}=477) \S\end{array}$ \\
\hline HAP MAS & $66.9^{*+}(19.5)$ & $75.7(6.2)$ & $84.8(7.8)$ \\
\% patients with HAP MAS $<5^{\circ}$ & $32 / 104(30.8)$ & na & na \\
\% patients with HAP MAS $<25^{\circ}$ & $44 / 104(42.3)$ & na & na \\
HAP AAS & $49.7^{*+}(22.9)$ & $71.6(7.1)$ & $83.2(7.8)$ \\
\% patients with HAP AAS $<5^{\circ}$ & $69 / 104(66.3)$ & na & na \\
\% patients with HAP AAS $<25^{\circ}$ & $83 / 104(79.8)$ & na & na \\
\hline
\end{tabular}

Data presented as mean (standard deviation) or number of patients (percentage)

${ }^{*} \mathrm{P}<.001$ vs elderly subjects; $+\mathrm{P}<.001$ vs healthy normal subjects

AAS, Adjusted Activity Score; HAP, Human Activity Profile; MAS, Maximal Activity Score; na, not available; § Fix A, Daughton D. Human Activity Profile(HAP) Manual. Odessa, FL, Psycological Assessment Resources Inc, 1986, tab. 12

Table 2. Barriers to physical activity (physical activity levels defined by AAS)

\begin{tabular}{lcccc}
\hline Barriers & $\begin{array}{c}\text { Patients, (104) } \\
\mathrm{n} \mathrm{( \% )}\end{array}$ & $\begin{array}{c}\text { Inactive (54) } \\
\mathrm{n}(\%)\end{array}$ & $\begin{array}{c}\text { Active, (50) } \\
\mathrm{n}(\%)\end{array}$ & $\mathrm{P}$ \\
\hline No place to exercise & $7(6.7)$ & $3(2.9)$ & $4(3.8)$ & 0.708 \\
No exercise partner & $15(14.4)$ & $11(1.6)$ & $4(3.8)$ & 0.096 \\
Fatigue on dialysis days & $59(56.7)$ & $33(31.7)$ & $26(25.0)$ & 0.460 \\
Fatigue on non-dialysis days & $20(19.2)$ & $8(7.7)$ & $12(11.5)$ & 0.348 \\
Pain on dialysis days & $30(28.9)$ & $19(18.3)$ & $11(10.6)$ & 0.205 \\
Pain on non-dialysis days & $23(22.2)$ & $14(13.5)$ & $9(8.7)$ & 0.461 \\
Lack of time on dialysis days & $34(32.7)$ & $14(13.5)$ & $20(19.2)$ & 0.187 \\
Lack of time on non-dialysis days & $15(14.4)$ & $8(7.7)$ & $7(6.7)$ & 1.000 \\
Lack of time because of too many & $15(14.4)$ & $8(7.7)$ & $7(6.7)$ & 1.000 \\
medical appointments & & & & \\
I don't want to & $44(42.3)$ & $25(24.0)$ & $19(18.3)$ & 0.511 \\
Feeling too old & $26(25.0)$ & $18(17.3)$ & $8(7.7)$ & 0.041 \\
Shortness of breath & $30(28.8)$ & $20(19.2)$ & $10(9.6)$ & 0.088 \\
Fear of getting hurt & $23(22.1)$ & $13(12.5)$ & $10(9.6)$ & 0.792 \\
Sadness & $52(50.0)$ & $33(31.7)$ & $19(18.3)$ & 0.031 \\
Feeling of helplessness & $46(44.2)$ & $28(26.9)$ & $18(17.3)$ & 0.153 \\
Inability to travel & $30(28.8)$ & $20(19.2)$ & $10(9.6)$ & 0.089 \\
Too many medical problems & $57(54.8)$ & $37(35.6)$ & $20(19.2)$ & 0.006 \\
Family concern & $25(24.1)$ & $14(13.5)$ & $11(10.6)$ & 0.812 \\
Physician concern & $2(2.0)$ & $1(1.0)$ & $1(1.0)$ & 1.000 \\
Chest pain & $12(11.6)$ & $11(10.6)$ & $1(1.0)$ & 0.009 \\
Amputation & $3(2.9)$ & $1(1.0)$ & $2(1.9)$ & 0.607 \\
Ulcers on legs and feet & $8(7.7)$ & $6(5.8)$ & $2(1.9)$ & 0.273 \\
\hline & & & & \\
& & & & \\
& & & &
\end{tabular}

were feeling to have too many medical problems (OR 3.27, 95\% CI 1.46 to $7.31 ; \mathrm{P}=0.004$ ), chest pain (OR 12.54, 95\% CI 1.55 to 101.11; P=0.018), sadness (OR 2.56, 95\% CI 1.16 to 5.65; $\mathrm{P}=0.020$ ), feeling too old (OR 2.63, 95\% CI 1.02 to 6.75 ; $\mathrm{P}=0.045$ ), and dyspnea (OR $2.35,95 \%$ CI 0.97 to $5.71 ; \mathrm{P}=0.058$ ). At multivariable analysis, after adjusting for age and sex, only feeling to have too many medical problems (OR 2.99, 95\% CI 1.27 to $7.07 ; \mathrm{P}=0.012$ ), chest pain (OR 10.78, 95\% CI 1.28 to 90.28; P=0.029) and sadness (OR 2.59, 95\% CI 1.10 to 6.09; $\mathrm{P}=0.030$ ) were independently associated with physical inactivity. 


\section{Kidney \\ Blood Pressure Research}

Table 3. Health personnel attitudes toward physical activity in ESRD patients

\begin{tabular}{rlcc}
\hline Item & Nephrologists & Dialysis nurses \\
\hline 1) & Physical inactivity (sedentary lifestyle) is an important health & $5 / 5$ & $24 / 24$ \\
& & \\
2) Insk in the general population. & $5 / 5$ & $24 / 24$ \\
3) & 5 Physical activity is beneficial for patients on dialysis & $23 / 24$ \\
4) I am concerned about the risks of exercise in patients on & $0 / 5$ & $3 / 24$ \\
$\quad$ dialysis & & \\
5) I believe that most dialysis patients would increase physical & $1 / 5$ & $13 / 24$ \\
$\quad$ activity if advised to do so & & \\
6) I have time to talk to dialysis patients about physical activity & $1 / 5$ & $2 / 24$ \\
7) I think dialysis patients are interested in the topic of physical & $1 / 5$ & $11 / 24$ \\
$\quad$ activity & & \\
8) I think exercise is important (or is as important as other & $0 / 5$ & $2 / 24$ \\
$\quad$ medical issues) & & \\
9) I think it is the role of the physician to counsel dialysis patients & $0 / 5$ & $7 / 24$ \\
$\quad$ about physical activity & & \\
10) I feel confident in my ability to discuss this topic with patients & $0 / 5$ & $8 / 24$ \\
11) Often ask & $4 / 5$ & $17 / 24$ \\
12) Often counsel & $5 / 5$ & $20 / 24$ \\
13) Often ask and counsel & $5 / 5$ & $19 / 24$ \\
Reserved to Nephrologists & & \\
14) Often prescribe & $4 / 5$ & - \\
15) Often provide written material & $3 / 5$ & - \\
16) Often refer & $3 / 5$ & - \\
17) Often provide equipment & $1 / 5$ & - \\
\hline
\end{tabular}

Attitude to physical activity and exercise counseling among nephrologists and dialysis nurses

Data on physical activity and exercise counseling rates among nephrologists and dialysis nurses are shown in Table 3.

Answers suggest that doctors and nurse are well aware of the risks associated with sedentarism in dialysis patients, and that they agree on the positive effects of physical activity. However, difficulties in increasing physical activity in dialysis patients are perceived, and are mainly attributed to the low compliance/interest by the patients toward exercise, as well as to the lack of time for exercise counseling by doctors and nurses.

\section{Discussion}

In the present single-center pilot study we evaluated the possible role of patient- and health personnel-related barriers in reducing the level of physical activity in a ESRD patient cohort on chronic dialysis in Italy.

Our results suggest that both types of barriers could play an important role in the pathogenesis of sedentarism typically observed in this clinical setting.

Available data from other countries, such as US, have documented that dialysis patients represent an highly inactive population [1], and that inactivity is associated with poor outcomes [4]. In the same patients many different clinical, social and psychological barriers to physical activity frequently coexist; in particular, lack of motivation, disease burden perception, and shortening of available time for exercise in dialysis days due to waiting times, treatment itself, and logistic problems, were among the most commonly indicated barriers [5-6]. Moreover, a low frequency of physical activity assessment and low rate of exercise 


\section{Kidney Blood Pressure Research}

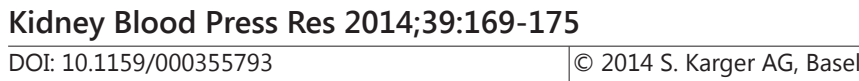

Published ontIne: July 29, 2014

www.karger.com/kbr

Fiaccadori/Sabatino/Schito et al.: Barriers to Physical Activity in Hemodialysis Patients

counseling by nephrologists has been also reported [7]. This latter attitude is surprising since, in the specific setting of ESRD, patients are usually seen thrice weekly by nephrologists and dialysis nurses, who often represent the main or the primary health provider for long period of times, and health personnel seems to be well aware of the importance of physical activity.

More than half of the patients enrolled in our study could be defined totally inactive, a finding similar to that obtained in US dialysis facilities [1]; many patients were only moderately active and only a minority was active. In our single-center population of Italian dialysis patients barriers to physical activity were also frequently observed. In fact, at least one barrier was reported in $96.1 \%$ of patients, with only $3 \%$ of patients perceiving only one barrier and almost 50\% having 5-9 barriers. Fatigue on dialysis days, perception of too many medical problems and sadness were reported by more than half of the patients; lack of motivation (42.3\%) and lack of time in dialysis days (32.7\%) were also common complaints. However, only perception of having too many medical problems, chest pain and sadness were independently associated with inactivity at multivariable analysis.

Besides their number, at least in part related to age, both the different characteristics of the barriers to physical activity and the diversity of the national health systems complicate the approach to overcome those barriers. For example, at least in theory, in Italy a free and widely covering national health system should facilitate the solving of some social and economic problems. However, as observed in this study, a number of important barriers cannot be considered strictly clinical or economic barriers and cannot be removed easily within the dialysis unit context, for example through the optimization of medical therapy and/ or dialysis schedule. Psychological barriers (for example motivation) or logistical barriers (availability of places to exercise, tutoring availability, etc) would require the involvement of other specific expert professionals, ideally working together with the dialysis team. Finally, some barriers to physical activity are likely to derive from attitudes and opinions of dialysis doctors and nurses. ESRD patients on dialysis typically develop a strong and long-lasting trust relationship with nephrologists and dialysis nurses. Consequently, as dialysis facility personnel usually represent the most important medical reference point, if nephrologists and nurses do not advise about physical activity, it is unlikely that patients will receive such counseling by other professionals. Available data suggest that exercise counseling rate is low among nephrologist, nothwithstanding the widespread awareness about both the high cardiovascular risk of these patients and the positive effects of physical activity. Lack of time, feeling non-confident in skills about exercise counseling, the belief that most dialysis patients would not increase physical activity even if advised to do so, and the opinion that dialysis patients are scarcely interested in the topic of physical activity and would hardly modify their level of physical activity are the most frequently reported barriers in the literature studies [7].

Also, our data highlight some controversial issues about attitudes of health personnel in dialysis units toward patients' physical activity. In fact, while nephrologists and nurses are fairly convinced of the risks associated with sedentarism in ESRD patients, and of the advantages of physical activity, they still believe that increasing physical activity in ESRD patients is exceedingly difficult. This belief is based mainly on a low compliance/interest to exercise by the patients, but also on a lack of time of doctors and nurses for counseling.

\section{Conclusion}

Physical activity level is low in ESRD patients on dialysis. Many coexisting factors are likely to concur in decreasing physical activity. Among them, different type of barriers are frequently present, either patient-specific but also related to attitudes and beliefs of doctors and nurses on exercise in ESRD patient. Addressing these issues may help in increasing the levels of physical activity in this population. 


\section{Kidney \\ Blood Pressure Research}

Fiaccadori/Sabatino/Schito et al.: Barriers to Physical Activity in Hemodialysis Patients

\section{Disclosure Statement}

The authors have no sponsorship or funding arrangements relating to their research, nor conflicts of interest.

\section{References}

-1 Johansen KL, Chertow GM, Kutner NG, Dalrymple LS, Grimes BA, Kaysen GA: Low level of self-reported physical activity in ambulatory patients new to dialysis. Kidney Int 2010;78:1164-1170.

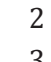
Johansen KL, Delgado C, Bao Y, Tamura MK: Frailty and dialysis initiation. Sem Dial 2013;26:690-696.

- 3 Painter P, Roshanvaran B: The association of physical activity and physical function with clinical outcomes in adults with chronic kidney disease. Curr Op Nephrol Hypert 2013;22:615-623.

-4 Johansen KL, Kaysen GA, Dalrymple LS, Grimes BA, Glidden DV, Anand S, Chertow GM: Association of Physical Activity with Survival among Ambulatory Patients on Dialysis: The Comprehensive Dialysis Study. Clin J Am Soc Nephrol 2013;8:248-253.

5 Delgado C, Johansen KL: Barriers to exercise partecipation among dialysis patients. Nephrol Dial Transpl 2012;27:1152-1157.

6 Zheng J, You LM, Lou TQ Chen NC, Lai DY, Liang YY, Li YN, Gu YM, Lv SF, Zhai CQ: Development and psychometric evaluation of the Dialysis Patient-perceived Exercise Benefits and Barriers Scale. Int J Nurs Studies 2010;47:166-180.

-7 Johansen KL, Sakkas GK, Doyle J, Shubert T, Dudley RA: Exercise counseling practices among nephrologists caring for patients on dialysis. Am J Kidney Dis 2003;41:171-178.

8 Johansen KL, Painter P, Kent-Braun JA, Ng AV, Carey S, Da Silva M, Chertow GM: Validation of questionnaires to estimate physical activity and functioning in end-stage renal disease. Kidney Int 2001;59:1121-1127.

-9 Davidson M, de Morton N: A Systematic review of the Human Activity Profile. Clin Rehab 2007;21:151-162. 